BULLETIN OF THE

AMERICAN MATHEMATICAL SOCIETY

Volume 77, Number 3, May 1971

\title{
CLASSIFICATION OF SYMBOL SPACES OF FINITE TYPE
}

\author{
BY R. F. WILLIAMS ${ }^{1}$ \\ Communicated by Stephen Smale, November 18, 1970
}

Introduction. Symbol spaces and their shift maps have a long history in dynamics beginning at the latest in 1921 with Morse [6]. Recently the work of Smale [10], Bowen-Lanford [3] and especially Bowen [1], [2], and Alekseev [0] shows that subshifts of finite type are crucial in the study of diffeomorphisms satisfying Smale's Axiom A. Thus the problem of classifying these elementary dynamical systems is basic to the geometric study of diffeomorphisms.

In this setting, Bowen-Lanford show how to attach a square matrix $A$ (of zeros and ones) to each shift of finite type. But this actually dates from the earlier papers [7], [8] of Parry on intrinsic Markov chains. The problem is to classify, up to conjugacy, the shift maps of a shift space of finite type. Obviously the number of periodic points of each period is an invariant. All of these numbers are contained in the zeta function, shown to be the reciprocal of $\operatorname{det}(I-t A)$ by Bowen-Lanford.

But the zeta function is not enough-see Example 3 below. Something like similarity $\left(A \sim P A P^{-1}\right)$ over the integers (actually positive integers) is necessary. The fact that this is in general not possiblethe inverse of a positive matrix, even if it exists and is integral, generally has negative entries-indicates the need for a more subtle equivalence relation.

We use shift equivalence (over $\boldsymbol{Z}^{+}$). This is the same type of equivalence which was used in an earlier paper [11] to classify onedimensional attractors. In fact, we feel that some sort of "shift equivalence" will be involved for any classification of basic sets of Axiom A diffeomorphisms. The recent work of Sinai [9] and Bowen [1], showing that each basic set is a quotient in a nice way of subshift of finite type, is a major step in their classification. I have just been informed that Alekseev has also proved this theorem.

AMS 1970 subject classifications. Primary 58F15, 58F20, 54H20.

Key words and phrases. Symbol space, shift of finite type, Bernoulli shift, intrinsic Markov chain, topological conjugacy, basic set, Axiom A, zeta function, shift equivalence, baker's map.

${ }^{1}$ We received partial support in this research from NSF Grant GP 9637. We would also like to thank C. Zeeman and L. Motchane for their hospitality at the University of Warwick, Mathematics Institute, and the Institut des Hautes Etudes Scientifiques, Bures-sur-Yvette, respectively. 
I would like to thank several colleagues for their generous help in conversations: Professors W. Parry, J. Guckenheimer, I. Herstein, Peter Walters, and especially Rufus Bowen who raised the basic question answered by Example 3 and Theorem $A$.

1. Basic definitions. Let $A$ be an $n \times n$ 0-1 matrix, i.e. each entry $A(i, j)$ is either 0 or 1 . Then the subshift of finite type determined by $A$ is the space of all doubly infinite sequences $\left(x_{i}\right)_{i \in Z}$ such that $x_{i}$ $=1,2, \cdots, n$ and $A\left(x_{i}, x_{i+1}\right)=1$ for each $i$, and will be denoted by $S(A) . S(A)$ is a metric space with the distance function,

$$
d(x, y)=\sum_{i=-\infty}^{\infty}\left(1-\delta_{x_{i} y_{i}}\right) 2^{-|i|},
$$

is compact and zero dimensional. There is the shift map $\hat{s}: S(A)$ $\rightarrow S(A)$ defined by $\hat{s}(x)=y$, where $y_{i}=x_{i+1}$ for all $i \in Z$. In the case $A(i, j)=1$ for all $i, j, S(A)$ is called the Bernoulli shift or full shift on $n$ symbols. For two square matrices $A, B$ with entries in $Z^{+}$one says $A$ is shift equivalent to $B$ (over $\left.\boldsymbol{Z}^{+}\right),\left(A \sim_{s} B\right.$ ) provided there are two rectangular matrices $R, S$ over $Z^{+}$and an integer $m$ such that $R A$ $=B R, S B=A S, S R=A^{m}$ and $R S=B^{m} . m$ is called the lag of the equivalence $R, S$. This is an equivalence relation (see [11]). Next define $A \sim{ }_{e} B$ provided $A=R S$ and $B=S R$ for rectangular matrices $R, S$ over $Z^{+}$. Making this relation transitive by allowing finite strings, one obtains an equivalence relation $\sim_{S}$ called strong shift equivalence.

Note that we do not require our matrices $A, B$, etc. to have only zeros and ones. Such a matrix determines a shift of finite type by "symbol splitting." Allowing entries $\geqq 2$ has several advantages: it lowers the dimension of many interesting examples, allows one to proceed without bothering to check inequality $\leqq 1$, and allows $A B$ whenever $A$ and $B$ are allowed.

Finally, two maps $f: X \rightarrow X$ and $g: Y \rightarrow Y$ are conjugate (or topologically conjugate) provided there is a homeomorphism $h: X \rightarrow Y$ such that

is commutative.

$$
\begin{aligned}
& X \stackrel{f}{\rightarrow} X \\
& h \downarrow \downarrow h \\
& Y \rightarrow Y \\
& g
\end{aligned}
$$

The relation between topological conjugacy of "shift maps" and shift equivalence was studied somewhat in [11]. An elementary argu- 
ment $[11,(4.6)$, p. 350$]$ is given to show $\sim_{8} \Leftrightarrow \sim_{S}$ in most categories. However, because of the special requirement of positivity a new proof is needed here for $\sim_{s} \Rightarrow \sim_{S}$ (the other direction is trivial). In fact, this argument constitutes the bulk of the paper. The fact that $A \sim{ }_{8} B \Rightarrow \zeta_{A}$ $=\zeta_{B}$ is elementary $[11,(4.8)$, p. 351$]$.

Finally, given $\sim_{s} \Rightarrow \sim_{S}$ half of Theorem A (below) is a consequence of the

Formula. For an elementary equivalence $A=S R, R S=B$ between two 0-1 matrices $A, B$, one has for each pair $(j, k)$ such that $A(j, k)=1$, a unique $l=l(j, k)$ such that $S_{j l} R_{l k}=1$, because $1=A(j, k)=\sum_{l} S_{j l} R_{l k}$. Then this elementary equivalence induces the conjugacy $\Phi: S(A)$ $\rightarrow S(B)$ where $[\Phi(x)]_{i}=l\left(x_{i}, x_{i+1}\right)$.

\section{Statement of results.}

TheOREm A. If $A$ and $B$ are square matrices over $\boldsymbol{Z}^{+}$, the shifts they determine are topologically conjugate iff $A$ and $B$ are shift equivalent over $\boldsymbol{Z}^{+}$.

THEOREM B. The Bernoulli shifts (full shifts) are the only shifts of finite type for which $1 / \zeta$ is linear.

THEOREM C. If $S(A)$ is topologically transitive, nonwandering and $\operatorname{det} A= \pm 1$, then $S(A)$ and $S(B)$ are topologically conjugate iff there is a matrix $R$ over $Z^{+}$such that $R A=B R$ and $\operatorname{det} R= \pm 1$.

THEOREM D. Given a shift equivalence between square matrices $A, B$ over $Z^{+}$, there is a finite procedure for constructing a conjugacy between $S(A)$ and $S(B)$.

Problem. Given matrices $A, B$, is there a finite procedure for deciding whether $A$ and $B$ are shift equivalent? If in addition $\operatorname{det} A$ $=\operatorname{det} B \neq 0$ ? Or if $A$ and $B$ are $2 \times 2$ or $\operatorname{det} A=\operatorname{det} B= \pm 1$ ?

The bulk of the paper consists of analysing conjugacy classes of "one sided shifts" (called baker's maps) $a: C(A) \rightarrow C(A)$ and their relation to the full shifts $\hat{a}: S(A) \rightarrow S(A)$ via inverse limits.

EXAMPLES. 1.

$$
\left(\begin{array}{ll}
1 & 1 \\
1 & 1
\end{array}\right) \sim_{s}(2)
$$

and they present the Bernoulli shift on 2 symbols.

2.

$$
\left(\begin{array}{rr}
10 & 2 \\
2 & 1
\end{array}\right) \sim\left(\begin{array}{ll}
9 & 4 \\
3 & 2
\end{array}\right)
$$


and $m$ can be taken to be 2 but not 1 .

3.

$$
\left(\begin{array}{ll}
4 & 1 \\
1 & 0
\end{array}\right) \text { and }\left(\begin{array}{ll}
3 & 2 \\
2 & 1
\end{array}\right)
$$

are not shift equivalent though they are similar if division by two is allowed. In particular their zeta functions are the same.

4.

$$
\left(\begin{array}{lll}
2 & 1 & 0 \\
1 & 1 & 1 \\
0 & 1 & 2
\end{array}\right)
$$

is not shift equivalent to any matrix with nonzero determinant.

5.

$$
\left(\begin{array}{lll}
2 & 1 & 0 \\
1 & 1 & 1 \\
0 & 1 & 2
\end{array}\right) \text { and }\left(\begin{array}{lll}
0 & 1 & 0 \\
0 & 2 & 1 \\
0 & 0 & 3
\end{array}\right)
$$

are not shift equivalent over $\boldsymbol{Z}^{+}$but they are similar over $\boldsymbol{Z}$.

Full proofs will appear elsewhere, [12].

\section{BIBLIOGRAPHY}

0. V. Alekseev, Quasirandom dynamical systems. I, II, III, Mat. Sb. 76 (118) (1968), 72-134 = Math. USSR Sb. 5 (1968), 73-128; ibid. 77 (119) (1968), 545-601 =ibid. 6 (1968), 505-560; ibid. 78 (120) (1969), 3-50=ibid. 7 (1969), 1-43.

1. R. Bowen, Markov partitions for Axiom A diffeomorphisms, Preprint, Math. Institute, University of Warwick; Amer J. Math. 42 (1970), 725-747.

2. - Topological entropy and Axiom A, Proc. Sympos. Pure Math., vol. 14, Amer. Math. Soc., Providence, R. I., 1970, pp. 23-42.

3. R. Bowen and O. Lanford, Zeta functions of restrictions of the shift transformation, Proc. Sympos. Pure Math., vol. 14, Amer. Math. Soc., Providence, R. I., 1970, pp. 43-50.

4. F. Gantmacher, The theory of matrices, GITTL, Moscow, 1953; English transl., vol. 2, Chelsea, New York, 1959. MR 16, 438; MR 21 \#6372c.

5. G. Hedlund, Endomorphisms and automorphisms of the shift dynamical system, Math. Systems Theory 3 (1969), 320-375.

6. M. Morse, Representation of geodesics, Amer. J. Math. 43 (1921), 33-51.

7. W. Parry, Entropy and generators in ergodic theory, Benjamin, New York, 1964.

8. - Intrinsic Markov chains, Trans. Amer. Math. Soc. 112 (1964), 55-66. MR 28 \#4579.

9. Ja. G. Sinal, Markov partitions and Y-diffeomorphisms, Funkcional. Anal. i Priložen. 2 (1968), no. 1, 64-89=Functional Anal. Appl. 2 (1968), 61-82. MR 38 \#1361. 
10. S. Smale, Diffeomorphisms with many periodic points, Differential and Combinational Topology (A Symposium in Honor of Marston Morse), Princeton Univ. Press, Princeton, N. J., 1965, pp. 63-80. MR 31 \#6244.

11. R. Williams, Classification of one-dimensional attractors, Proc. Sympos. Pure Math., vol. 14, Amer. Math. Soc., Providence, R. I., 1970, pp. 341-361.

12. - Classification of symbol spaces of finite type, Preprint, Northwestern University, Evanston, Ill., 1970.

NORTHWESTERN UNIVERSITY, Evanston, ILLINOIS 60201

Institut des Hautes Etudes Scientifiques, Bures-sur-Yvette, France 